\title{
A Survey on Different Recommendation Techniques
}

\author{
Neha Shrivastava ${ }^{1}$, Surendra Gupta ${ }^{2}$ \\ ${ }^{1}$ Research Scholar, Department of Computer Engineering, Shri Govindram Seksaria Institute of Technology and \\ Science (S.G.S.I.T.S), Indore, India, shrivastavaneha0209@gmail.com \\ ${ }^{2}$ Professor, Department of Computer Engineering Shri Govindram Seksaria Institute of Technology and Science \\ (S.G.S.I.T.S), Indore, India, sgupta@ @sgsits.ac.in
}

Received Date : October 02, 2021 Accepted Date : October 23, 2021 Published Date : November 07, 2021

\begin{abstract}
Recommender systems are used to provide recommendation or suggestions on services and items to the users. It provides suggestions which based on prediction. Prediction plays an important role in recommender systems while making the recommendation for users, prediction ensures the quality of suggestions for their users. Recommendation system used in various applications such as e-commerce websites, social Applications, search engines, or for daily decisions that we need to make. To generate prediction for recommender systems many techniques have been proposed. The goal of the paper is to provide a general overview on various recommendation techniques. It covers the working of different recommendation techniques along with its classification and also their advantages and disadvantages.
\end{abstract}

Key words: Collaborative filtering, Content-based filtering, Hybrid filtering.

\section{INTRODUCTION}

Recommender System plays an important role in World Wide Web. On the web, a number of options are available to generate results for a problem, but there is need to select, prioritize and effectively use the particular information to generate the solution to a problem for users. Recommendation systems provide solution to this problem by searching through huge amount of information or data and provide a solution to a user on their personalized content. It produces accurate recommendations from the collection of users for items that might be liked by the user. Recommendation systems used in every analytics technique to make users daily life easier by selecting in which they are interested like as product, news, entertainment, social networks, food items and so on. There is explosive growth of the data generation in the world day by day and it is become difficult to everyone for searching the items due to large amount of data. So, the recommendation system is used to reduce searching time of users. The efficiency of recommendation provided to user depends on accuracy of recommendations. There are many recommendation algorithms are existing to save the time of user in selecting items. The working of recommendation system models is based on collaborative filtering based, content-based and hybrid based.

\section{RELATED WORK}

Through the years, many recommendation systems had been advanced the usage of collaborative, content based and hybrid filtering strategies. these models have been implemented the use of numerous machine learning algorithms. various writers presented distinctive recommendation systems and their strategies to enhance the suggestions or recommendations for users. This section incorporates a quick mention about some techniques proposed via specific authors. In [1] the authors propose a prediction method in recommendation systems and also discuss their benefits and characteristics. In [2] the authors proposed a movie recommendation system using user based and item-based technique on movie lens dataset and analyze the performance of both the algorithm. In [3] the authors propose a recommendation system. The authors have used the weighted Pearson similarity function to improve the performance of user based and item based collaborative filtering model. In [4] the authors implemented a project for movie recommendation system that depends on content based filtering algorithm and collaborating filtering algorithm. authors also discussed hybrid filtering in brief. In [5] the authors propose an implementation of movie recommendation system using collaborative filtering method. The proposed model is built using Apache Mahout library based on the Apache software foundation. Authors propose a content-based filtering model for recommendation system [6].

In [7] the authors propose a collaborative filtering method for recommendation generation and discuss the problems present in a collaborative filtering method. The authors implemented improved hybrid algorithm for user. Authors propose a recommendation system on hybrid technique. To enhance the performance of prediction in recommendation system authors 
used feature selection process and apply naïve Bayesian classifier [8]. In [9] the authors proposed a hybrid model for recommender system. The proposed model works on feedback system in which feedback gathered from the public platforms and from the users. In [10] the authors propose a survey on collaborative filtering technique, discuss issues, limitations, benefits present in a collaborative filtering technique also explain hybrid approach. Writers proposed a comparison of collaborative filtering method and Hybrid method. The comparison of both the approach have been implemented for a movie recommender system [11]. In [12] the authors propose a survey on recommendation system that combines advantages, disadvantages, algorithm used for recommendation system. The authors also propose web page hybrid collaborative filtering model that used pattern finding algorithms. In [13] the authors propose a comparative study on clustering methods, which are K-Means clustering and Agglomerative hierarchical clustering. On the basis of studies, we say that, collaborative filtering has become observed to be one of the famous used processes to construct recommendation systems.

\section{TYPES OF RECOMMENDATION TECHNIQUES}

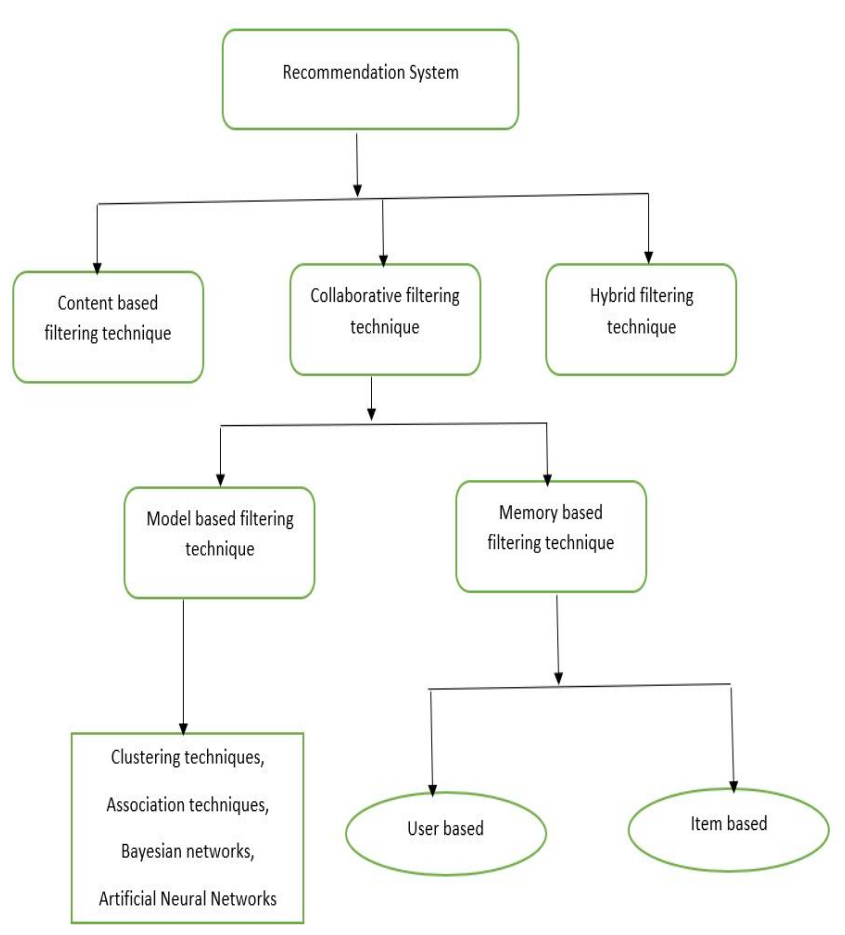

Figure 1: Structure of Recommendation System Methods

\subsection{Content Based Filtering Technique}

This technique is based on domain and it is used the attributes of content to produce the predictions for users. Content based technique mostly used in social networks, web-based services and publications. Content-based filtering technique work on the user profiles and their characteristics to generate recommendations. All the data is used for content-based filtering is gathered from description and characteristics of particular item contents along with the user's preference which user had already made in the past. Content based recommender model uses data of items based on the correlation between the user choices and items content. It requires facts such as what the user might interested or what the user previous history. This technique is totally depending on user's previous performance or feedback and what user likes or dislikes. Content based filtering uses different types of algorithms to generate the similarity among the contents so that it will generate worthful suggestions.

TF/IDF- Content-based filtering technique widely used tf-idf algorithm to generate content-based profile of user. Tf-idf creates user profile on a weighted vector of item features. The weights associated with each item feature shows the importance of feature to the user and calculated individually rated content vectors using average value of the rated items. Figure 2 shows the process flow of content-based filtering technique -

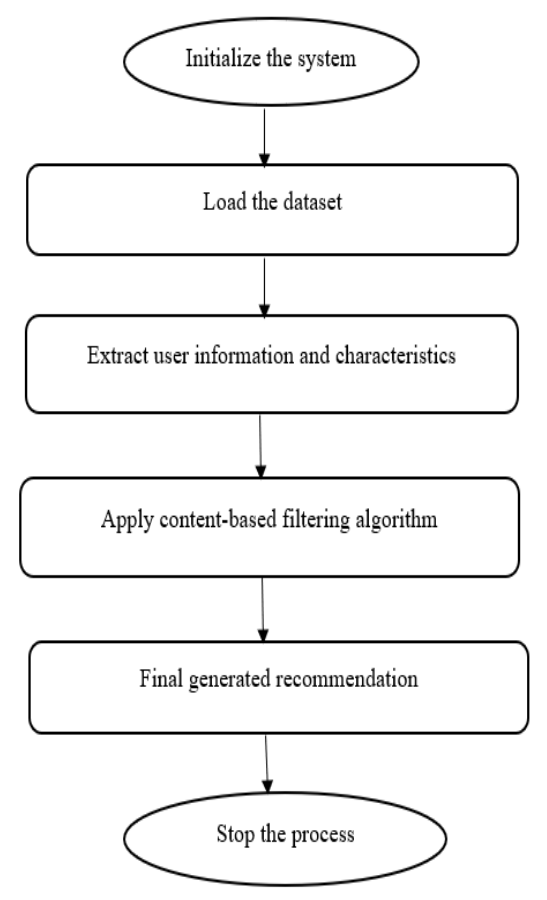

Figure 2: Flow diagram of Content Based Filtering Technique

\subsection{Collaborative Based Filtering Technique}

Collaborative filtering methods based on the user interests. The main purpose of this method is to find the neighbors of active users whose choices are similar to active user. It 
basically suggests the items to users that have same kind of preferences. This technique works on the assumption of users that define users have the same preferences in future as they have previously. It's collected the data of users based on their choices and preferences and selects other users who have same choice and likeness by computing similarity that present in between their preferences. Collaborative filtering works on memory based and model-based kinds. Figure 3 shows the process flow of Collaborative filtering technique-

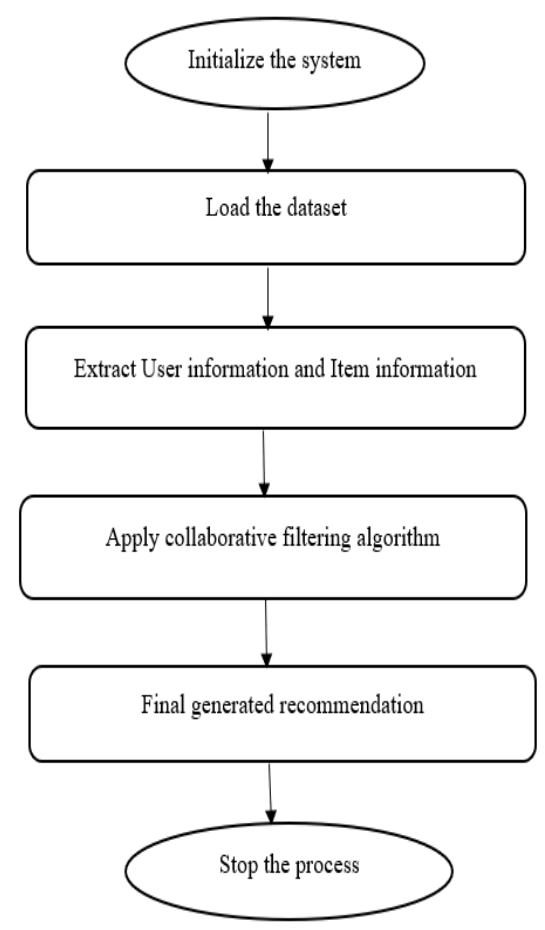

Figure 3: Flow diagram of Collaborative Based Filtering Technique

\subsubsection{Memory based collaborative filtering}

Memory based filtering technique rely on the calculating similarity between user and items. In this filtering technique items are already rated by the users. This technique focuses on searching the neighbors of users and then calculating their preferences. Memory based filtering works on two ways user based collaborative filtering and item based collaborative filtering.

\section{A. User Based collaborative Filtering}

This filtering technique works with the similarity in between users and it depended on the neighbors of user. In this technique similarity is calculated between users over a same item. This filtering technique make the prediction that depends on ratings given to the items by the different users who have same kind of choices with the target user. In user-based model for user and item we calculate the ratings of all the neighbors of target user, for the target object after which test the similarity between neighborhood users and target users.

Steps that involved in checking the similarity between target user and neighborhood users for the target item are -

1 Create the user-item matrix for users and items.

2 Calculate the similarity between the active or target user and neighborhood users using Pearson- Correlation or Cosine-Similarity.

3 Similarity Coefficient is calculated using average rating of top $\mathrm{N}$ users who gave the rating to target item.

4 The ratings are generated by computation of weighted average of ratings.

\section{B. Item Based Collaborative Filtering}

Item based collaborative filtering compute the similarity in among items or products. In this technique, items are selected which are much like the item selected by the neighbors of users. This filtering determines, how similar a selected item is with the target item. In item-based model, for a targeted item, compute all the neighborhood items that are similar to target item.

Steps that involved in checking the similarity between target item and neighborhood items are -

1 Create the user- item matrix for users and items.

2 Calculate the similarity available in between the target item and neighborhood items by using Pearson- Correlation or Adjusted Cosine-Similarity.

3 Similarity Coefficient is calculated using the average rating of nearest neighbor items.

4 The ratings are generated by computation of weighted average of ratings.

\subsubsection{Model based collaborative filtering}

Model based collaborative filtering technique used past ratings to prepare a system that allows to enhance the overall working of Collaborative filtering method. Machine learning algorithm are used to build a model and these models help to recommend a set of items quickly because it uses pre-computed model. To identify the relationships between items this technique uses different rules.

A. Association rule: Association rules are data mining methods which is used to discover hidden pattern. It extracts regulations that are expecting the occurrence of an object primarily focused on the availability of different objects in a transaction. 
B. Clustering: Clustering is a well-established unsupervised data mining approach that group data points based on similarities. It is a technique in which a group of similar data objects combined together to form a new cluster. Clustering results in minimization of the dimensionality of data set. Clustering algorithm tries to divide data objects into sub-clusters to obtained the meaningful group. In this technique data objects are considered as an input and as an output a number of clusters are produced. In produced data objects those objects are similar are belong to the same group called cluster and they are different from the objects of another group or cluster. Clustering strategies are used in different areas inclusive of, pattern recognition, image processing, statistical data analysis and knowledge discovery.

C. Decision tree: Decision tree is a classification technique belongs to the tree graphs method. In this algorithm data objects are split into a number of simpler subsets. These subsets are producing solutions which are easy to interpret. Decision tree model created by examine a collection of training examples where class labels are present and then it applies on the classify unseen examples.

D. Artificial Neural network: ANN is a set of multiple related units known as artificial neurons and these nodes are organized in layers in systematic approaches. The connection forms in among neurons have some weight with them that shows the amount of influence one neuron has on another neuron.

\subsection{Hybrid Filtering Technique}

Hybrid based filtering method works on the combination of different recommendation approaches to construct better system enhancement to generate worthful recommendation. Hybrid approach were proposed to overcome and reduces the drawbacks of original recommendation techniques. It improves the quality of recommendation and offered more effective, better recommendations than a single algorithm.

In hybrid model, limitations of single algorithm or model can be reduced by another algorithm so by means of the use of a couple of recommendation algorithm can reduce the imperfections of particular algorithm in a combined model.
Hybrid technique can be classified into three types and these are integrated, flow, and parallel type.

1. In integrated kind of hybrid technique, some algorithms are mixed to generate a complete system via using distinctive recommendation techniques.

2. In flow kind of hybrid technique, recommendation process is split into many sub-parts in order to generate a final result by using variety of recommendation algorithms.

3. In parallel type, several recommendation algorithms are combined by using some mixing mechanism to generate the final result and these mechanisms are mixed hybrid, weighted hybrid, and switching hybrid strategies. Mixed hybrid combines the results of several approaches to generate recommendation result. Weighted hybrid using fixed linear equation to add the two-result list of recommendation approaches. Switching hybrid systems takes the multiple recommenders and selects only the best one to generate recommendations.

To construct hybrid model, Combination of different algorithms can be used in following manners:

Implementing each algorithm separately and combining their final result, make use of content-based methods in collaborative approach, apply some collaborative filtering methods in content-based approach, constructing an integrated recommendation system that brings collectively each approach. 
Neha Shrivastava et al., International Journal of Emerging Trends in Engineering Research, 9(11), November 2021, 1367 - 1372

\section{COMPARATIVE ANALYSIS OF RECOMMENDATION TECHNIQUES}

Table 1: Comparative Analysis

\begin{tabular}{|c|c|c|c|}
\hline $\begin{array}{l}\text { Recommendation } \\
\text { Technique }\end{array}$ & Description & Advantages & Disadvantages \\
\hline Content based filtering & $\begin{array}{l}\text { Recommendation is depending } \\
\text { on facts of object contents and } \\
\text { their characteristics. }\end{array}$ & $\begin{array}{l}\text { - New items do not require } \\
\text { ratings for making } \\
\text { recommendation } \\
\text { - Works with lesser data }\end{array}$ & $\begin{array}{l}\text { - Need to deep } \\
\text { knowledge and } \\
\text { detailed description } \\
\text { of item features. } \\
\text { - Limited content } \\
\text { analysis } \\
\text { - Incapable to find users } \\
\text { preferences towards } \\
\text { products }\end{array}$ \\
\hline Collaborative filtering & $\begin{array}{l}\text { Recommendation is based on } \\
\text { collecting user preferences and } \\
\text { their past behavior. }\end{array}$ & $\begin{array}{l}\text { - Does not require domain } \\
\text { knowledge } \\
\text { - Other users scores are used }\end{array}$ & $\begin{array}{l}\text { - Require rating data } \\
\text { - Suffers from cold } \\
\text { start, data sparsity, } \\
\text { scalability problem }\end{array}$ \\
\hline Hybrid filtering & $\begin{array}{l}\text { Recommendation is primarily } \\
\text { based on combination of content } \\
\text { based and collaborative filtering } \\
\text { or individual approach of each } \\
\text { technique. }\end{array}$ & $\begin{array}{l}\text { Provide more accurate and effective } \\
\text { recommendation }\end{array}$ & $\begin{array}{l}\text { Inappropriate combination of } \\
\text { recommendation techniques } \\
\text { leads to poor result }\end{array}$ \\
\hline
\end{tabular}

\section{CONCLUSION}

Recommendation system have the ability to produce accurate recommendation to the users which are helpful to reduce the search timing of users. This surveyed paper is based on the techniques used for the working of recommendation system. From the distinctive feature of this paper, it could be concluded that the simple collaborative filtering technique stays the identical in every strategy mentioned on this paper. The working behind the recommendation model is purely based on mentioned filtering techniques in this survey paper. These filtering techniques uses different approaches to build a good recommendation system.

\section{REFERENCES}

1. F.O. Isinkaye, Y.O. Folajimi, B.A. Ojokoh "Recommendation systems: Principles, methods and evaluation" November 2015 Journal of Science direct.

2. Garima Gupta, Rahul Katarya "Recommendation Analysis on Item-based and User Based Collaborative" 2019 International Conference on Smart Systems and Inventive Technology (ICSSIT).
3. Sravan Kumar Gadekula, Udai Pratap Rao, Ram Krishan Vyas, Amrutha Lakshmi Dontula, Suraj V Gaikwad,

"Improved Pearson Similarity for Collaborative Filtering Recommendation on System", 2019 6th International Conference on Computing for Sustainable Global Development (INDIACom).

4. Rajeev Kumar, Guru Basava, Felicita Furtado1, "An Efficient Content, Collaborative - Based and Hybrid Approach for Movie Recommendation Engine", International Journal of Trend in Scientific Research and Development (IJTSRD).

5. Ching-Seh Mike Wu, Deepti Garg, Unnathi Bhandary, "Movie Recommender System Using Collaborative Filtering", 2018 IEEE 9th International Conference on Software Engineering and Service Science (ICSESS).

6. R. Van Meteren, and M. Van Someren, "Using content-based filtering for recommendation", In Proceedings of the Machine Learning in the New Information Age: MLnet/ECML2000 Workshop, vol. 30, pp. 47-56, May 2000.

7. Junrui Yang, Cai Yang, Xiaowei Hu, "A Study of Hybrid Recommendation Algorithm Based on User", 2016 8th International Conference on Intelligent Human-Machine Systems and Cybernetics (IHMSC). 
8. S. Sathiya Devi, G. Parthasarathy, "A Hybrid Approach for Movie Recommendation System Using Feature Engineering", 2018 Second International Conference on Inventive Communication and Computational Technologies (ICICCT).

9. Kavinkumar V., Rachamalla Rahul Reddy, Rohit Balasubramanian, Sridhar M., Sridharan K., D. Venkataraman, "A Hybrid Approach for Recommendation System with Added Feedback Component", 2015 International Conference on Advances in Computing, Communications and Informatics (ICACCI).

10. Prateek Parhi, Ashish Pal, Manuj Aggarwal, "A Survey of Methods of Collaborative Filtering Techniques", 2017 International Conference on Inventive Systems and Control (ICISC).

11. Noor Ifada, Triyani Fatchur Rahman, Mochammad Kautsar Sophan, "Comparing Collaborative Filtering and Hybrid based Approaches for Movie Recommendation", 2020 6th Information Technology International Seminar (ITIS).

12. Paritosh Nagarnaik, A. Thomas, "Survey on Recommendation System Methods", 2015 2nd International Conference on Electronics and Communication Systems (ICECS).

13. Karthikeyan B., Dipu Jo George, G. Manikandan, Tony Thomas, "A Comparative Study on K-Means Clustering and Agglomerative Hierarchical Clustering", International Journal of Emerging Trends in Engineering Research, Volume 8. No. 5, May 2020. 